\title{
Risk of keratinocyte carcinoma among patients with hidradenitis suppurativa
}

\author{
S. Ashrafzadeh ${ }^{1,2}$, Y. Kim ${ }^{1,2}$, G.A. Peters ${ }^{1}$, H. Lee ${ }^{3}$, M.M. Asgari $^{1,2}$ \\ ${ }^{1}$ Department of Dermatology, Massachusetts General Hospital, Boston, MA, USA \\ ${ }^{2}$ Department of Population Medicine, Harvard Medical School and Harvard Pilgrim Healthcare \\ Institute, Boston, MA, USA \\ ${ }^{3}$ Biostatistics Center, Massachusetts General Hospital, Boston, MA, USA
}

Dear Editor, Hidradenitis suppurativa (HS), also known as acne inversa, is a chronic inflammatory disorder of the pilosebaceous units. ${ }^{1}$ To date, studies on the risk of keratinocyte carcinoma (KC), defined as basal cell carcinoma (BCC) and squamous cell carcinoma (SCC), among HS patients have been limited. ${ }^{2-5}$ We performed a case-control study comparing the incidence of BCC and SCC in HS patients (cases) to that in acne vulgaris patients (controls), which is also an inflammatory disorder of the pilosebaceous unit. $^{1}$

Using an age-, sex-, and race-matched 1:2 case-control study design, patients with at least one International Classification of Disease, $9^{\text {th }}$ or $10^{\text {th }}$ Revision (ICD-9/10) code for HS or acne vulgaris were identified in our institutional database of seven hospitals (Partners Healthcare, 2000-2019). HS and acne diagnosis codes were validated by reviewing electronic medical records of a random $10 \%$ sample of each group for descriptions of characteristic lesions arising within relevant anatomic sites, supplemented with pathology reports, when available. Patients diagnosed initially as having HS but later diagnosed with an alternate dermatologic disorder (e.g. furuncles) were not classified as HS cases.

KCs were identified using pathology reports and ICD-9/10 codes and were confirmed using pathology record review or clinical note review. We determined both overall and incident (i.e. after HS/acne diagnosis) BCC and SCC risk by performing multivariate logistic regression adjusted for age at time of HS/acne diagnosis, sex, race, BMI, smoking, chronic immunosuppressive conditions known to increase KC risk (solid organ transplantation, HIV infection, chronic lymphocytic leukemia), ${ }^{6}$ and number of dermatologist visits per year (a measure of surveillance bias) using Stata 15.0 (College Station, TX). The Partners IRB approved this study.

The study sample included 13,812 patients (HS $n=4,604$, acne $n=9,208$ ). The mean age was 43.7 ( $\pm 15.4 \mathrm{SD}$ ) years; $74.7 \%$ were female; and $59.3 \%$ were white. The positive predictive value of HS and acne diagnosis codes were $92 \%$ and $95 \%$, respectively. Approximately

Corresponding Author: Maryam M. Asgari, MD, MPH, masgari@partners.org.

Conflicts of Interest: None declared. 
$2.1 \%$ of HS patients and $4.3 \%$ of acne patients developed BCCs whereas $2.3 \%$ of HS and $2.1 \%$ of acne patients developed SCCs (Table 1). Of 104 SCCs in the HS cohort, $4.8 \%$ (5/104, all males) developed at HS sites on the buttocks or perineum. Adjusted odds ratios (OR) of overall BCC (OR 0.40, 95\% CI 0.31-0.52) and incident BCC (OR 0.48, 95\% CI $0.35-0.67)$ were significantly lower in the HS cohort. In contrast, adjusted odds of overall SCC (OR 1.00, 95\% CI 0.76-1.32) and incident SCC (OR 0.98, 95\% CI 0.68-1.42) were similar between groups. HS patients developed their first SCC at a younger age than controls (58.9 years vs. 62.2 years, $\mathrm{P}=0.026$ ) but developed their first $\mathrm{BCC}$ at a similar age to controls ( 58.8 years vs. 55.9 years, $\mathrm{P}=0.052$ ). Risk of $\mathrm{BCC}$ and $\mathrm{SCC}$ stratified by sex did not differ between cases and controls.

Similar to our findings, studies suggest that SCCs arising at HS sites predominantly occur in men and in the buttocks or perineum. ${ }^{3,5}$ For KCs located at any site, a study on hospitalized, Swedish patients found increased SCC risk associated with HS; BCC risk was not examined. ${ }^{2}$ However, the study did not account for SCC risk factors or surveillance bias, and it only reported a SCC incidence rate of 24/100,000 person-years among 2,119 HS patients in contrast to our study's SCC incidence rate of 278/100,000 person-years among 4,604 HS patients, which is more consistent with published SCC disease estimates, ${ }^{7}$ suggesting potential incomplete ascertainment of SCCs. ${ }^{2}$

Strengths of our study include adjusting for some known KC risk factors and dermatologist surveillance, examining a large and racially diverse patient sample, and quantifying both BCC and SCC risk. Study limitations include inability to account for certain risk factors (e.g. sun exposure) and use of a single institutional data source.

Our results suggest that HS patients have reduced risk of BCC, but similar risk of SCC, compared with acne patients. However, HS patients develop SCCs at a younger age than acne patients. Our findings may reflect different mechanistic pathways involved in disease pathogenesis, differential exposure to sunlight and other environmental factors that affect skin cancer risk, or distinct treatment paradigms as HS patients are often exposed to immunosuppressive regimens. ${ }^{8}$ Chronic inflammation may also contribute to the younger age of SCC development among HS patients. ${ }^{4}$ Further studies are needed to replicate our findings in other populations. Our findings have implications for clinicians caring for $\mathrm{HS}$ patients, who may benefit from increased awareness of the risk of SCC arising at a younger age among HS patients when performing skin examinations.

\section{Acknowledgments}

Funding: This work was supported by the National Institute of Arthritis and Musculoskeletal and Skin Diseases (K24 AR069760 to MA) and the National Cancer Institute (R01 CA231264 and R01 CA241623 to MA).

\section{References}

1. Pink A, Anzengruber F, Navarini AA. Acne and hidradenitis suppurativa. Br J Dermatol. 2018;178(3):619-631. doi:10.1111/bjd.16231 [PubMed: 29380349]

2. Lapins J, Ye W, Nyrén O, Emtestam L. Incidence of Cancer Among Patients With Hidradenitis Suppurativa. Arch Dermatol. 2001;137(6):730-734. doi:10-1001/pubs.ArchDermatol.ISSN-0003-987x-137-6-dst00132 [PubMed: 11405761] 
3. Jourabchi N, Fischer AH, Cimino-Mathews A, Waters KM, Okoye GA. Squamous cell carcinoma complicating a chronic lesion of hidradenitis suppurativa: a case report and review of the literature. Int Wound J. 2017;14(2):435-438. doi:10.1111/iwj.12671 [PubMed: 27681476]

4. Chapman S, Delgadillo D III, Barber C, Khachemoune A. Cutaneous squamous cell carcinoma complicating hidradenitis suppurativa: a review of the prevalence, pathogenesis, and treatment of this dreaded complication. ACTA Dermatovenerol Alp PANNONICA Adriat. 2018;27(1):25-28. doi:10.15570/actaapa.2018.5 [PubMed: 29589641]

5. Lavogiez C, Lavogiez C, Delaporte E, et al. Clinicopathological Study of 13 Cases of Squamous Cell Carcinoma Complicating Hidradenitis Suppurativa. Dermatology. 2010;220(2):147-153. doi:10.1159/000269836 [PubMed: 20029163]

6. Xu MJ, Lazar AA, Garsa AA, et al. Major prognostic factors for recurrence and survival independent of the American Joint Committee on Cancer eighth edition staging system in patients with cutaneous squamous cell carcinoma treated with multimodality therapy. Head Neck. 2018;40(7):1406-1414. doi:10.1002/hed.25114 [PubMed: 29524273]

7. Elliott BM, Douglass BR, McConnell D, Johnson B, Harmston C. Incidence, demographics and surgical outcomes of cutaneous squamous cell carcinoma diagnosed in Northland, New Zealand. N Z Med J. 2018;131(1475):61-68.

8. Frew JW, Vekic DA, Woods J, Cains GD. A systematic review and critical evaluation of reported pathogenic sequence variants in hidradenitis suppurativa. Br J Dermatol. 2017;177(4):987-998. doi:10.1111/bjd.15441 [PubMed: 28278367] 
Table 1.

Overall and incident basal cell carcinoma and squamous cell carcinoma risk among patients with hidradenitis suppurativa (cases) compared with acne vulgaris (controls).

\begin{tabular}{|c|c|c|c|c|c|}
\hline Variable & HS $(n=4,604)$ & Acne $(\mathrm{n}=9,208)$ & Crude OR (95\% CI) & Adjusted $\mathrm{OR}^{a}(95 \% \mathrm{CI})$ & P-value \\
\hline \multicolumn{6}{|l|}{ Basal Cell Carcinoma (BCC) } \\
\hline Overall BCC Cases, n (\%) & $95(2.1 \%)$ & $400(4.3 \%)$ & $0.46(0.37-0.58)$ & $0.40(0.31-0.52)$ & \\
\hline Age in years, mean (SD) $b$ & $58.8(12.6)$ & $55.9(12.7)$ & & & 0.052 \\
\hline Male, n (\%) & $30(31.6 \%)$ & $138(34.5 \%)$ & $0.42(0.28-0.63)$ & $0.35(0.22-0.55)$ & \\
\hline Female, n $(\%)$ & $65(68.4 \%)$ & $262(65.5 \%)$ & $0.49(0.37-0.64)$ & $0.43(0.32-0.59)$ & \\
\hline White, $\mathrm{n}(\%)$ & $90(94.7 \%)$ & $384(96.0 \%)$ & $0.45(0.36-0.57)$ & $0.40(0.31-0.52)$ & \\
\hline Non-white, $\mathrm{n}(\%)^{c}$ & $5(5.3 \%)$ & $16(4.0 \%)$ & $0.62(0.23-1.70)$ & $0.51(0.18-1.46)$ & \\
\hline Incident BCC Cases, n (\%) & $60(1.3 \%)$ & $245(2.7 \%)$ & $0.48(0.36-0.64)$ & $0.48(0.35-0.67)$ & \\
\hline Age in years, mean (SD) $b$ & $61.8(13.1)$ & $58.9(12.0)$ & & & 0.103 \\
\hline Male, n (\%) & $21(35.0 \%)$ & $89(36.3 \%)$ & $0.46(0.29-0.75)$ & $0.40(0.23-0.70)$ & \\
\hline Female, n (\%) & $39(65.0 \%)$ & $156(63.7 \%)$ & $0.49(0.35-0.70)$ & $0.53(0.36-0.78)$ & \\
\hline White, $\mathrm{n}(\%)$ & $56(93.3 \%)$ & $236(96.3 \%)$ & $0.46(0.35-0.62)$ & $0.47(0.34-0.66)$ & \\
\hline Non-white, $\mathrm{n}(\%)^{c}$ & $4(6.7 \%)$ & $9(3.7 \%)$ & $0.89(0.27-2.88)$ & $0.78(0.22-2.80)$ & \\
\hline \multicolumn{6}{|c|}{ Squamous Cell Carcinoma (SCC) } \\
\hline Overall SCC Cases, n (\%) & $104(2.3 \%)$ & $195(2.1 \%)$ & $1.07(0.84-1.36)$ & $1.00(0.76-1.32)$ & \\
\hline Age in years, mean (SD) $b$ & $58.9(13.5)$ & $62.2(11.1)$ & & & 0.026 \\
\hline Male, n (\%) & $40(38.5 \%)$ & $87(44.6 \%)$ & $0.92(0.63-1.34)$ & $0.85(0.54-1.33)$ & \\
\hline Female, n (\%) & $64(61.5 \%)$ & $108(55.4 \%)$ & $1.19(0.87-1.62)$ & $1.09(0.76-1.56)$ & \\
\hline White, n (\%) & $96(92.3 \%)$ & $181(92.8 \%)$ & $1.06(0.83-1.37)$ & $1.04(0.78-1.40)$ & \\
\hline Non-white, $\mathrm{n}(\%){ }^{c}$ & $8(7.7 \%)$ & $14(7.2 \%)$ & $1.14(0.48-2.73)$ & $0.75(0.30-1.88)$ & \\
\hline Incident SCC Cases, n (\%) & $55(1.2 \%)$ & $117(1.3 \%)$ & $0.94(0.68-1.30)$ & $0.98(0.68-1.42)$ & \\
\hline Age in years, mean (SD) $b$ & $61.9(13.1)$ & $62.0(11.9)$ & & & 0.932 \\
\hline Male, n (\%) & $25(45.5 \%)$ & $54(46.2 \%)$ & $0.92(0.57-1.49)$ & $0.92(0.52-1.62)$ & \\
\hline Female, n (\%) & $30(54.5 \%)$ & $63(53.8 \%)$ & $0.95(0.62-1.47)$ & $0.99(0.60-1.62)$ & \\
\hline White, $\mathrm{n}(\%)$ & $49(89.1 \%)$ & $106(90.6 \%)$ & $0.92(0.66-1.30)$ & $1.03(0.69-1.52)$ & \\
\hline Non-white, $\mathrm{n}(\%)^{c}$ & $6(10.9 \%)$ & $11(9.4 \%)$ & $1.09(0.40-2.95)$ & $0.75(0.26-2.15)$ & \\
\hline
\end{tabular}

${ }^{a}$ Overall BCC and SCC risk adjusted for age at time of HS or acne vulgaris diagnosis, race, sex, BMI, smoking, immunosuppression (defined as having an organ transplant, chronic lymphocytic leukemia, or HIV infection), and average number of dermatology visits per year. Incident BCC and SCC risk adjusted for above mentioned variables as well as history of prior KC.

${ }^{b}$ Age at first pathologically-verified diagnosis, p-value calculated using two-sample t-test.

${ }^{c}$ Non-white group includes Black, Hispanic, Asian, Pacific Islander, and Other/Unknown races. In both the HS and acne vulgaris cohorts, approximately $19.4 \%$ were Black; $8.8 \%$ were Hispanic; and $12.5 \%$ were Asian, Pacific Islander, or Other/Unknown. 